\title{
Reviews of effectiveness of training after spinal cord or traumatic brain injury
}

Spinal cord injury (SCI) and traumatic brain injury (TBI) have a devastating impact on those who sustain such injuries, as well as on communities and families. Ensuring that post-injury treatment is as effective as possible is crucial to the successful rehabilitation and quality of life of those who sustain such injuries.

Two new reviews have recently been published in the Cochrane Library (www. cochrane.org) examining training options after $\mathrm{SCI}^{1}$ and $\mathrm{TBI}{ }^{2}$

The first of these reviews examines the effectiveness of approaches to regaining walking function after SCI. ${ }^{1}$ The extent of disability after SCI varies greatly, depending on the severity and location of the injury and which nerve fibers are damaged. The resulting neurological deficit can be temporary or permanent, complete or incomplete; one of the primary goals for people with SCI is to improve locomotor function-that is, the ability to walk. Traditional treatments, compensation-based strategies (such as brace walking), are commonly used to facilitate walking, but do not result in the recovery of walking ability as was known before the injury.

Newer treatment options have been introduced in recent years for locomotor rehabilitation of people with some motor function. Such options may involve treadmill training with and without body weight support, robotic-assisted gait training, and functional electrical stimulation. These treatments aim to achieve restoration and recovery of walking.

The review examines the effectiveness and acceptability of locomotor training (defined as the repetitive practice of complex gait cycles-for example, treadmill-based walking) after SCI. The reviewers included randomized controlled trials (RCT) that compared locomotor training with any other exercise that has the goal of improving walking function after SCI, or with a no-treatment control group. Four RCTs involving 222 patients were included. Two studies investigated the robotic-assisted device "Lokomat" as one of the experimental interventions, and all four studies investigated bodyweight-supported treadmill training with manual guidance without any robotic device.

The review authors found that there were no significant differences between the different types of locomotor training for improving walking function in people with SCI. They commented that the insufficient evidence was mostly due to the small number of trials and small sample sizes of participants. A key recommendation of the review was that further multicenter RCTs with larger sample sizes be undertaken. Furthermore, the reviewers concluded that research should define subpopulations of people with SCI to find out who is benefiting most from which locomotor training approaches and at which recovery stage. Importantly, the reviewers stated that there was a need for research reports to describe the complete intervention strategy rather than just the modality of treatment, progression of therapy, and the role of the therapist.

Fitness training for cardiorespiratory conditioning after TBI was the focus of another recent review published in the Cochrane Library from the Injuries Group. ${ }^{2}$ TBI is the leading cause of longterm disability in children and young adults. As it typically occurs in the younger population, people who have sustained such an injury will be expected to survive for many years, and it is therefore important to optimize the health of people with TBI throughout their life. Reduced fitness is a common problem after TBI. Clinically, fitness training is used to address this problem. This review aimed to examine fitness training as an intervention for cardiorespiratory fitness in people who have sustained a TBI.

Reviewers included six randomized trials in the review, including 303 participants. The participants were primarily male, in their mid-thirties, who had sustained a severe TBI. Three of the six studies indirectly assessed change in cardiorespiratory fitness after fitness training using the peak power output obtained during cycle ergometry. Cardiorespiratory fitness was improved after fitness training in one study (mean difference $59 \mathrm{~W}, 95 \%$ CI 24 to 94), but there was no significant improvement in the other two studies. Studies could not be pooled for the primary outcome because of diversity with regard to the interventions, time after injury, and the outcome measures.

The authors concluded that there is insufficient evidence to draw any definitive conclusions about the effects of fitness training on cardiorespiratory fitness. However, they concluded that fitness training appears to be a safe and accepted intervention for people with TBI, but that more adequately powered and well-designed studies are required to determine the effects across a range of outcome measures.

These two reviews highlight once more the need for rigorously conducted and adequately powered research studies to evaluate interventions that are currently in use or under development. Although these reviews did not reveal any harmful effects of the interventions under examination, this is always a possibility that must be considered. The opportunity cost for interventions that have no or minimal effect is also important, given the limited resources available for healthcare.

The Cochrane Injuries Group completes reviews on effectiveness of interventions for the prevention, treatment, and rehabilitation of traumatic injury. If you are interested in learning more about the group contact the review group coordinator, Emma Sydenham, at Emma. Sydenham@1shtm.ac.uk or see the Cochrane Injuries Group website at http://injuries.cochrane.org. Reviews are published in the Cochrane Library, available at www.cochrane.org.

R Ivers

The George Institute for International Health, Sydney, NSW 2050, Australia; rivers@george.org.au

Competing interests: None.

\section{REFERENCES}

1. Mehrholz J, Kugler J, Pohl M. Locomotor training for walking after spinal cord injury. Cochrane Database Syst Rev 2008, Issue 2. Art. No.: CD006676 DOI: 10.1002/14651858.CD006676.

2. Hassett LM, Moseley AM, Tate R, et al. Fitness training for cardiorespiratory conditioning after traumatic brain injury. Cochrane Database Syst Rev 2008, Issue 2. Art. No.: CD006123 D0I: 10.1002/ 14651858.CD006123. 